\title{
Natural Stimulus Responsive Scaffolds/Cells for Bone Tissue Engineering: Influence of Lysozyme upon Scaffold Degradation and Osteogenic Differentiation of Cultured Marrow Stromal Cells Induced by CaP Coatings
}

\author{
Ana M. Martins, B.S., ${ }^{1-3}$ Quynh P. Pham, Ph.D., ${ }^{3}$ Patrícia B. Malafaya, Ph.D., ${ }^{1,2}$ \\ Robert M. Raphael, Ph.D., ${ }^{3}$ F. Kurtis Kasper, Ph.D., ${ }^{3}$ \\ Rui L. Reis, Ph.D., D.Sc., ${ }^{1,2}$ and Antonios G. Mikos, Ph.D. ${ }^{3}$
}

This work proposes the use of nonporous, smart, and stimulus responsive chitosan-based scaffolds for bone tissue engineering applications. The overall vision is to use biodegradable scaffolds based on chitosan and starch that present properties that will be regulated by bone regeneration, with the capability of gradual in situ pore formation. Biomimetic calcium phosphate $(\mathrm{CaP})$ coatings were used as a strategy to incorporate lysozyme at the surface of chitosan-based materials with the main objective of controlling and tailoring their degradation profile as a function of immersion time. To confirm the concept, degradation tests with a lysozyme concentration similar to that incorporated into $\mathrm{CaP}$ chitosan-based scaffolds were used to study the degradation of the scaffolds and the formation of pores as a function of immersion time. Degradation studies with lysozyme $(1.5 \mathrm{~g} / \mathrm{L})$ showed the formation of pores, indicating an increase of porosity ( $\sim 5-55 \%$ up to 21 days) resulting in porous threedimensional structures with interconnected pores. Additional studies investigated the influence of a $\mathrm{CaP}$ biomimetic coating on osteogenic differentiation of rat marrow stromal cells (MSCs) and showed enhanced differentiation of rat MSCs seeded on the CaP-coated chitosan-based scaffolds with lysozyme incorporated. At all culture times, CaP-coated chitosan-based scaffolds with incorporated lysozyme demonstrated greater osteogenic differentiation of MSCs, bone matrix production, and mineralization as demonstrated by calcium deposition measurements, compared with controls (uncoated scaffolds). The ability of these CaP-coated chitosan-based scaffolds with incorporated lysozyme to create an interconnected pore network in situ coupled with the demonstrated positive effect of these scaffolds upon osteogenic differentiation of MSCs and mineralized matrix production illustrates the strong potential of these scaffolds for application in bone tissue engineering strategies.

\section{Introduction}

$\mathbf{T}$

ISSUE ENGINEERING STRATEgIES include two general categories: (1) the use of acellular matrices (artificial scaffolds or decellularized tissues), which depend upon the natural ability of the body to regenerate for proper orientation and direction of new tissue growth, and (2) the use of scaffolds with cells. ${ }^{1,2}$ The typical strategy involves the use of a porous scaffold, which serves as a transplantation vehicle for cultured cells and a template to guide tissue regeneration, thereby playing an important role in transform- ing the cell-seeded scaffold into a new tissue. To allow a high density of colonizing cells and to promote neovascularization when implanted in vivo, the scaffolds should have high porosity, large surface area, mechanical properties and pore sizes appropriate for the application, and a highly interconnected pore structure. ${ }^{1}$ In addition, biocompatibility and biodegradability are also required properties. Bone regeneration is a complex series of events involving proliferation and differentiation of mesenchymal stem cells into osteoblasts, bone matrix production, and mineralization. ${ }^{3}$

\footnotetext{
${ }^{1} 3 \mathrm{~B}$ 's Research Group-Biomaterials, Biodegradables and Biomimetics, Department of Polymer Engineering, University of Minho, Headquarters of the European Institute of Excellence on Tissue Engineering and Regenerative Medicine, Guimarães, Portugal.

${ }^{2}$ PT Government Associated Laboratory, IBB-Institute for Biotechnology and Bioengineering, Guimarães, Portugal.

${ }^{3}$ Department of Bioengineering, Rice University, Houston, Texas.
} 
As extensively described in the literature, scaffold porosity is inversely related to the mechanical properties of the scaffold; thus, it is necessary to balance the mechanical needs of the particular tissue that is going to be replaced and the scaffold porosity sufficient to allow tissue growth. ${ }^{4,5}$ Therefore, this work proposes the use of nonporous scaffolds composed of an in vivo responsive material that will be regulated by bone regeneration, with gradual in situ pore formation and consequent scaffold resorption. ${ }^{6}$ This novel approach includes the use of smart and stimulus responsive scaffolds that might be intelligent enough to control their degradation profile as a function of implantation time and to induce osteogenic differentiation of marrow stromal cells (MSCs).

Chitosan has been considered to be a promising material for tissue engineering applications. ${ }^{7-9}$ It is a linear copolymer of N-acetyl-D-glucosamine and D-glucosamine, derived from the deacetylation of chitin. The deacetylation degree measures the percentage of glucosamine units in the polymer chain and influences its physicochemical properties such as solubility, crystallinity, swelling behavior, and biological properties, ${ }^{10}$ such as osteogenesis enhancement. ${ }^{11-13}$ The Nacetylglucosamine present in chitosan is also responsible for nitric oxide production by rat peritoneal exudate macrophages $^{14}$ at reasonable levels in terms of inflammatory response but very effective in terms of wound tissue repair. ${ }^{15}$ It has also been demonstrated that chitosan is degraded in vitro and in vivo by lysozyme, ${ }^{15-17}$ an enzyme ubiquitous in the human body. Moreover, hydrolytic actions of lysozyme are able to activate macrophages and to promote collagen deposition. The biocompatibility of chitosan might be attributed to its structural similarity to glycosaminoglycan, which is a major component of the extracellular matrix (ECM) of bone and cartilage. $^{12}$

Starch was used as a component of the scaffolds presented in this study as a sacrifice material that will be easily degraded by several enzymes occurring in the human body. ${ }^{6}$ Several in vitro and in vivo studies have shown that starchbased biomaterials are biocompatible $\mathrm{e}^{18-20}$ and biodegradable. ${ }^{6,21,22}$ The main enzymes involved in starch degradation are $\alpha$-amylases, $\beta$-amylases, $\alpha$-glucosidases, glucoamylases, and other debranching enzymes (pullulanase and isoamylase). ${ }^{23,24}$ The enzymatic degradation products of starch hydrolysis are mainly glucose, maltose, and dextrin. ${ }^{24}$

A potential method to enhance the bone bonding, osteoconductive, and/or osteoinductive capacity of chitosan and starch-based scaffolds is to apply on the surface of the threedimensional (3D) structures a calcium phosphate $(\mathrm{CaP})$ biomimetic coating with a composition similar to the major inorganic component of bone, hydroxyapatite. In addition, biomimetic $\mathrm{CaP}$ coatings have been considered as potential carriers of bioactive proteins without compromising their activity, as they are generated under physiological conditions ( $\left.\mathrm{pH} 7.4,37^{\circ} \mathrm{C}\right) .^{22,25-28}$ It has been shown that different proteins can be directly integrated into the structure of $\mathrm{CaP}$ coatings without compromising their activity, and their release is mainly controlled by the dissolution mechanism of the coating. ${ }^{22,25-27}$ In this sense, biomimetic coatings were used to tailor the material surface properties or to directly enhance cell adhesion, proliferation, and osteogenic differentiation. Moreover, $\mathrm{CaP}$ coatings are used as a means to functionalize biomaterials with biological affinity and protein adsorption properties, as previously mentioned. ${ }^{28}$ The incorporation of different enzymes into $\mathrm{CaP}$ coatings, prepared on the surface of scaffolds by means of a biomimetic coating technique, seems to be a very interesting strategy to control their degradation rate. In this work, lysozyme was incorporated into the coatings with the aim of developing a self-regulating degradable material with a capacity for gradual in situ pore formation. In addition, this system can simultaneously exhibit osteoinductive and osteoconductive properties in the sense that it can act as a carrier system for the controlled release of multiple biologically active proteins. In the past decade, a large number of publications showed osteoinduction by $\mathrm{CaP}$ coatings in several animal models. ${ }^{29,30}$ Recent research has shown that the biological performance of osteoinductive biomaterials, namely, on new bone growth orthotopically, is significantly better than nonosteoinductive biomaterials. $^{30}$

The present study reports the use of $\mathrm{CaP}$-coated chitosanbased scaffolds with incorporated lysozyme. This study was designed to answer the following questions: (1) How does lysozyme influence the formation of pores in chitosan scaffolds, using a similar concentration to the one incorporated into CaP coatings? (2) Does this concentration of lysozyme allow the formation of interconnected pores? (3) Are CaPcoated chitosan-based scaffolds able to enhance osteogenic differentiation of rat MSCs compared with uncoated (without $\mathrm{CaP}$ coating) scaffolds?

\section{Materials and Methods}

\section{Materials}

In this work, degradable scaffolds based on chitosan and corn starch were developed. Two different compositions were prepared using a precipitation $\operatorname{method}^{7}$ : chitosan $(\mathrm{Ch})$ and chitosan/starch (CS) scaffolds. Briefly, chitosan was dissolved in acetic acid $1 \%(\mathrm{v} / \mathrm{v})$ to obtain a $5 \%(\mathrm{w} / \mathrm{v})$ solution. Then, using the same procedure, another formulation was prepared with the following ratio: 60/40 chitosan/starch. The chitosan and chitosan/starch solutions were cast into molds and frozen $\left(-18^{\circ} \mathrm{C}\right)$ overnight. $^{6}$ They were then immersed in a precipitation solution $\left(25 \%(\mathrm{v}) \mathrm{NaOH} 1 \mathrm{M}\right.$ and $\left.75 \%(\mathrm{v}) \mathrm{Na}_{2} \mathrm{SO}_{4} 0.5 \mathrm{M}\right)$ overnight, adapted from Tuzlakoglu et al. ${ }^{7}$ After precipitation, the samples were washed several times with distilled water to remove excess salt from the precipitation solution. After this procedure, two other formulations were prepared based on biomimetic coating methodology, ${ }^{31,32}$ consisting of an impregnation of the materials with bioactive glass called Bioglass $^{\circledR}$ (45S5) supplied by NovaMin Technology (Alachua, FL) followed by an immersion in a simulated body fluid (SBF, $37^{\circ} \mathrm{C}$, pH 7.4), presenting ionic concentrations similar to human blood plasma. Briefly, chitosan (Ch) and chitosan/starch (CS) scaffolds were immersed in a wet bed of bioglass for $6 \mathrm{~h}$ in a rotator. After that, the scaffolds were immersed in simulated body fluid (1.0 SBF) solution with lysozyme $(1 \mathrm{~g} / \mathrm{L})$ for 7 days at $37^{\circ} \mathrm{C}$ (nucleation stage). This stage allows for the formation of $\mathrm{CaP}$ nuclei. After the nucleation stage, all samples were washed with distilled water and immersed in a concentrated simulated body fluid solution (1.5 SBF) for 7 days at $37^{\circ} \mathrm{C}$ to enhance CaP nuclei growth. Two different samples were obtained after the biomimetic coating technique: $\mathrm{CaP}$ chitosan scaffolds with incorporated lysozyme $(\mathrm{CaP} / \mathrm{Ch}+$ lysozyme) and $\mathrm{CaP}$ chitosan/starch scaffolds with incorporated lysozyme (CaP/CS + lysozyme). ${ }^{33}$ 


\section{Degradation studies}

Degradation studies were performed in triplicate by incubating the chitosan scaffolds $(\mathrm{Ch})$ in phosphate-buffered saline (PBS) solution ( $\mathrm{pH} 7.4$ ) with lysozyme (1.5 g/L), a concentration similar to the ones incorporated into $\mathrm{CaP}$ biomimetic coatings, at $37^{\circ} \mathrm{C}$ up to 21 days. Lysozyme solution was changed every 7 days. At the end of the degradation period, the samples were removed, washed with distilled water, and dried in a graded series of ethanol for later calculation of weight loss. The porosity, pore interconnectivity, pore size, and 3D structures of dry chitosan scaffolds were analyzed before and after degradation by microcomputed tomography $(\mu-\mathrm{CT})$.

\section{Microcomputed tomography}

The scaffolds were analyzed using $\mu$-CT before and after degradation with lysozyme for the different periods of immersion $(0,3,14$, and 21 days). $\mu$-CT was carried out using a Skyscan 1072 (Skyscan, Kontich, Belgium) (pixel size resolution of $6.59 \mu \mathrm{m}$ ), with X-ray source settings of $40 \mathrm{keV}$ and $250 \mu \mathrm{A}$. The data sets were acquired over a rotation range of $180^{\circ}\left(0.45^{\circ}\right.$ rotation step $)$ and reconstructed with software (NRecon v1.4.3; SkyScan). Representative data sets were segmented with a dynamic threshold of 40-255 and were used for morphometric analysis (CT Analyser, v1.5.1.5; SkyScan) and to build the 3D models (ANT 3D creator, v2.4; SkyScan). The morphometric analysis included porosity, pore interconnectivity, mean pore size, and mean fiber thickness. Three-dimensional virtual models of representative regions in the bulk of the scaffolds were created, visualized, and registered using both image processing software packages to access the morphological changes after degradation (CT Analyser and ANT 3D creator). The interconnectivity was quantified as the accessible void fraction over a range of minimum connection sizes using an analysis software tool (CT Analyser, v1.5.1.5; SkyScan). A 3D shrinkwrap process was performed to shrink the outside boundary of the volume of interest (VOI) in a scaffold through any openings whose size was equal to or larger than $53 \mu \mathrm{m}$. Any void space connected via interconnects below this size was considered to be inaccessible, ${ }^{34}$ based upon reported minimum pore interconnection sizes for bone tissue ingrowth. ${ }^{35,36}$ Interconnectivity was calculated as follows ${ }^{37}$ :

$$
\text { Interconnectivity }=\left(\mathrm{V}_{\mathrm{t}}-\mathrm{V}_{\mathrm{sw}}\right) /\left(\mathrm{V}_{\mathrm{t}}-\mathrm{V}_{\mathrm{m}}\right) \times 100 \%
$$

where $V_{\mathrm{t}}$ is the total volume of the VOI, $V_{\mathrm{sw}}$ is the VOI volume after $3 \mathrm{D}$ shrink-wrap processing, and $V_{\mathrm{m}}$ is the volume of scaffold material.

The wall thickness was defined as the pore wall thickness and was quantified with $\mu-\mathrm{CT}$ analysis based upon structural indices using a software tool (CT Analyser, v1.5.1.5; SkyScan). Specifically, quantitative analysis of the pore wall thickness was retrieved from the 3D analysis using structure thickness parameters.

\section{MSCs isolation}

Rat MSCs were obtained from femurs and tibias of 4144-day-old male Wistar rats (Charles River Laboratories, Wilmington, MA) using the method described by Maniato- poulos et al. ${ }^{38}$ The harvested cells were cultured until confluence in a humidified atmosphere with $5 \% \mathrm{CO}_{2}$ at $37^{\circ} \mathrm{C}$. Nonadherent cells were removed after 1 and 3 days. Upon reaching confluence, the cells were trypsinized, centrifuged, and resuspended in a known amount of medium and $10 \%$ dimethyl sulfoxide to obtain a final concentration of $3 \times 10^{6}$ cells $/ \mathrm{mL}$. This cell suspension was then aliquoted into cryotubes for storage in liquid nitrogen ( $1 \mathrm{~mL} /$ cryotube).

\section{Cell seeding}

Chitosan-based scaffolds with $6 \mathrm{~mm}$ diameter and thickness of $0.8-1.0 \mathrm{~mm}$ were press fit into 6-mm-diameter cassettes and covered with complete osteogenic medium [ $\alpha$-MEM supplemented with $10 \%$ fetal bovine serum from Cambrex/BioWhittaker (Walkersville, MD), $50 \mu \mathrm{g} / \mathrm{mL}$ gentamicin, $100 \mu \mathrm{g} / \mathrm{mL}$ ampicilin, $10 \mathrm{mM}$ fungizone, $50 \mu \mathrm{g} / \mathrm{mL}$ L-ascorbic acid, $0.01 \mathrm{M} \beta$-glycerophosphate, and $10^{-8} \mathrm{M}$ dexamethasone] overnight. After 1 day, each scaffold was seeded with 280,000 cells contained in $100 \mu \mathrm{L}$ of complete osteogenic media in accordance with previous studies. ${ }^{39,40}$ Three hours after cell seeding, $10 \mathrm{~mL}$ of complete osteogenic media was added to each well of a six-well plate. After $24 \mathrm{~h}$ of attachment, the scaffolds were placed in new six-well plates for 8, 16, and 21 days with complete osteogenic medium. Media were changed every 3 days. Each group consisted of $n=6$ samples.

At the end of each culture period, samples were removed from the cassettes and rinsed with PBS solution. Three samples from each group were stored at $-20^{\circ} \mathrm{C}$ in $\mathrm{ddH}_{2} \mathrm{O}$ until analyses were performed. One of the remaining three samples was immersed in PBS with calcein-AM for $45 \mathrm{~min}$ for observation under confocal microscopy, and the other two samples were fixed with $2.5 \%$ glutaraldehyde for Fourier transformed infrared spectroscopy (FTIR) analysis and for observation under scanning electron microscopy (SEM).

\section{DNA analysis}

Measurement of the cell number in chitosan-based scaffolds was assessed from cell lysates using the Picogreen dsDNA Quantification Kit according to the instructions of the manufacturer (Molecular Probes, Eugene, OR). All standards and samples were prepared in triplicate. The fluorescence at $545 \mathrm{~nm}$ was read on a plate reader (FLx800; Bio-Tek Instruments, Winooski, VT) to determine DNA concentrations per scaffold. The cellularity of each scaffold was calculated by correlation with the DNA concentrations measured from a known number of MSCs.

\section{Alkaline phosphatase activity measurements}

Alkaline phosphatase (ALP) activity assays were performed on cell lysates using an established protocol. ${ }^{39}$ All reagents were purchased from Sigma (St. Louis, MO). All samples were prepared in triplicate and compared against $p$ nitrophenol standards. The reaction was stopped by adding $100 \mu \mathrm{L}$ of $0.3 \mathrm{~N} \mathrm{NaOH}$. Absorbance was read at $405 \mathrm{~nm}$ on a plate reader (PowerWave $\times 340$; Bio-Tek) to determine the ALP activity per scaffold.

\section{Calcium deposition measurement}

Calcium deposition measurements were made in triplicate. CaP-coated chitosan-based scaffolds with incorporated 
lysozyme without cells at the end of each time period were used as controls to determine the calcium content of the coating. Then, these values were subtracted from the calcium content obtained by CaP-coated chitosan-based scaffolds with incorporated lysozyme cultured with cells to normalize the data. Calcium deposition was evaluated using a calcium assay from Diagnostic Chemicals (Oxford, CT). After ALP and DNA assays were completed, a volume of $1 \mathrm{~N}$ acetic acid equal to the remaining volume was added to each well (for a final concentration of $0.5 \mathrm{~N}$ ), and the plate was placed on an orbital shaker overnight. A volume of $300 \mu \mathrm{L}$ of calcium assay reagent was added to $20 \mu \mathrm{L}$ of sample solution in 96-well plates. All samples were assessed in triplicate. To generate a standard curve, serial dilutions of $\mathrm{CaCl}_{2}$ were prepared $(0-50 \mu \mathrm{g} / \mathrm{mL})$. Absorbance was read at $650 \mathrm{~nm}$ with a spectrophotometer plate reader (PowerWave $\times 340$; Bio-Tek). The calcium deposition in each scaffold is reported as $\mu \mathrm{g} \mathrm{Ca}{ }^{2+}$.

\section{Microscopy analysis}

After each time period, chitosan-based scaffolds were removed from the cassettes, washed with PBS, and stained with calcein-AM (Molecular Probes) according to the manufacturer's instructions. After $45 \mathrm{~min}$ immersion in PBS with calcein-AM, images were recorded using a laser scanning confocal microscope with a $10 \times$ APO objective (Zeiss LSM 510 Axiovert; Carl Zeiss, Oberkochen, Germany). Samples for SEM were washed twice in PBS and fixed with $2.5 \%$ glutaraldehyde for $30 \mathrm{~min}$. Then, the scaffolds were washed with PBS and dehydrated in a graded series of ethanol, and dried after immersion in hexamethyldisilazane.

\section{FTIR with attenuated total reflectance}

Chitosan-based scaffolds before (control samples) and after 16 days of culture were analyzed by FTIR-attenuated total reflectance (ATR). The scaffolds were fixed in $2.5 \%$ glutaraldehyde, dehydrated in a graded series of ethanol, and dried at room temperature. All spectra were recorded using 64 scans and $4 \mathrm{~cm}^{-1}$ in an FTIR spectrophotometer (IR Prestige-21, Fourier Transformer infrared spectrophotometer; Shimadzu, Kyoto, Japan).

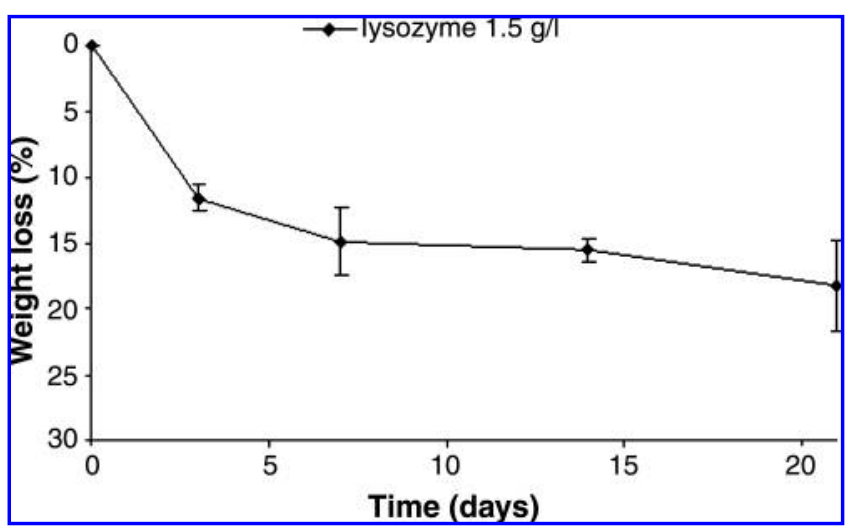

FIG. 1. Weight loss of chitosan (Ch) scaffolds after 21 days of immersion in PBS with lysozyme $(1.5 \mathrm{~g} / \mathrm{L})$ solution. Chitosan scaffolds were gradually degraded as a function of time in lysozyme solution $(1.5 \mathrm{~g} / \mathrm{L})$.

\section{Statistics}

Results of DNA, ALP, and calcium assays are expressed as mean \pm standard deviation with $n=3$ for each group. Statistically significant differences were determined using Student's $t$-test multiple comparison procedure at a confidence interval of $95 \%(p<0.05)$.

\section{Results and Discussion}

\section{Degradation studies}

The degradation of chitosan in the human body has been reported to be carried out by lysozyme. ${ }^{16,17}$ Previous studies have identified the optimal concentration of lysozyme for the degradation of chitosan-based scaffolds in vitro to be $1.5 \mathrm{~g} / \mathrm{L} .{ }^{33}$ Accordingly, a lysozyme concentration of $1.5 \mathrm{~g} / \mathrm{L}$ was used in the present study for optimal degradation of the chitosan-based scaffolds, as well as to approximate the

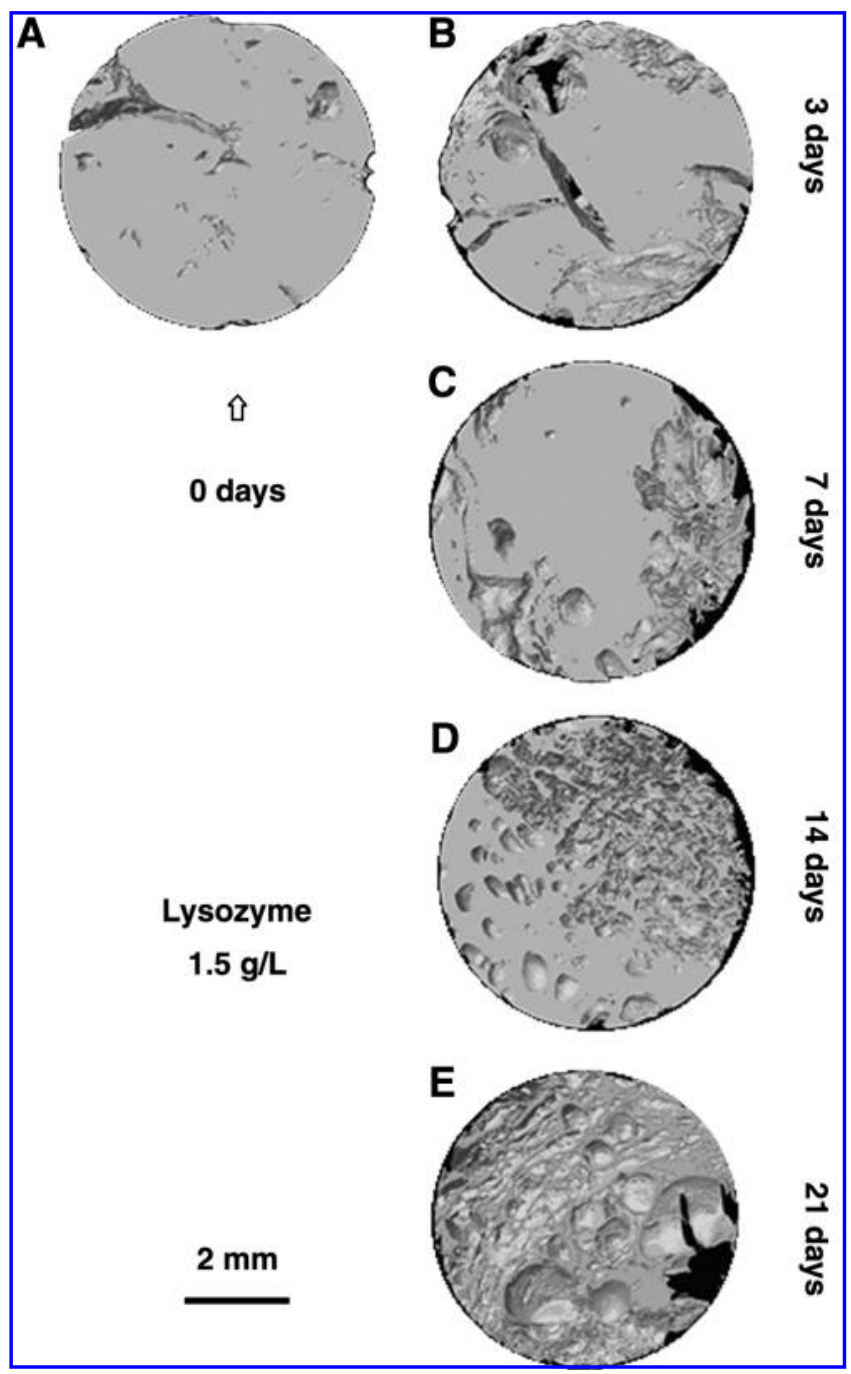

FIG. 2. Images of chitosan (Ch) scaffolds obtained by $\mu-C T$ : before degradation (A) and after 3 days (B), 7 days (C), 14 days (D), and 21 days (E) of degradation with lysozyme $(1.5 \mathrm{~g} / \mathrm{L})$. Lysozyme solution induced degradation and enhanced subsequent formation of pores in chitosan scaffolds as a function of immersion time. 

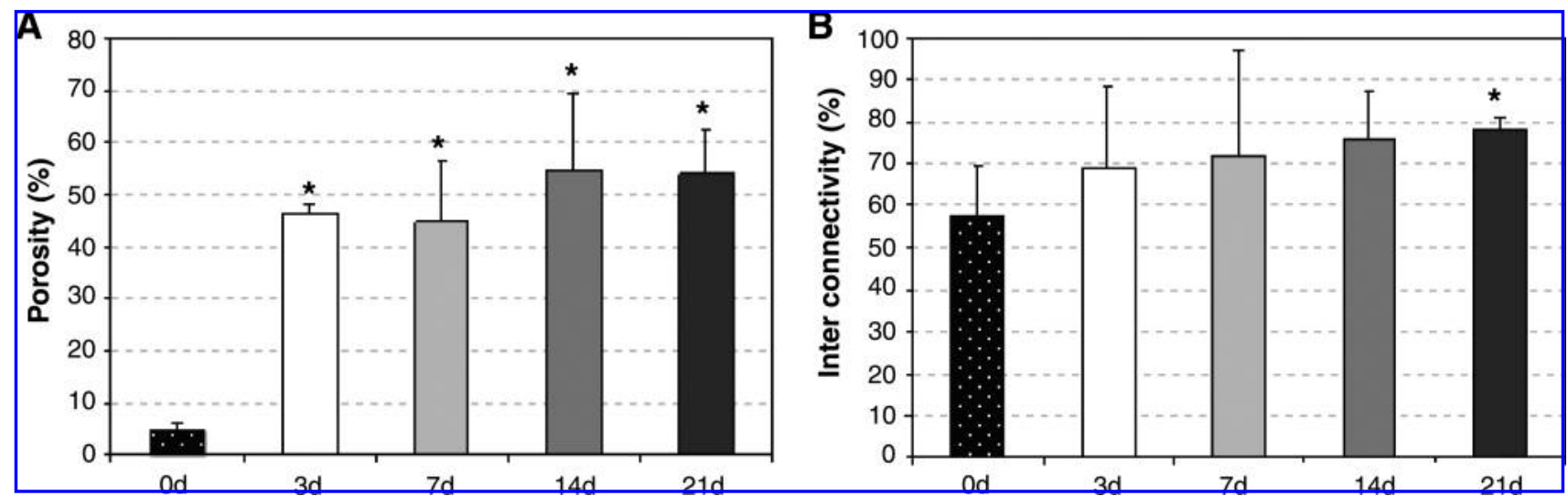

FIG. 3. Porosity (A) and interconnectivity (B) of chitosan scaffolds as a function of immersion time with lysozyme (1.5 g/L). Results are expressed as means \pm standard deviation with $n=3$ for each bar. Asterisk $\left({ }^{*}\right)$ indicates a significant difference $(p<0.05)$ as a function of time compared with control samples. Immersion of chitosan scaffolds in lysozyme solution increased the porosity and pore interconnectivity of the scaffolds with time.
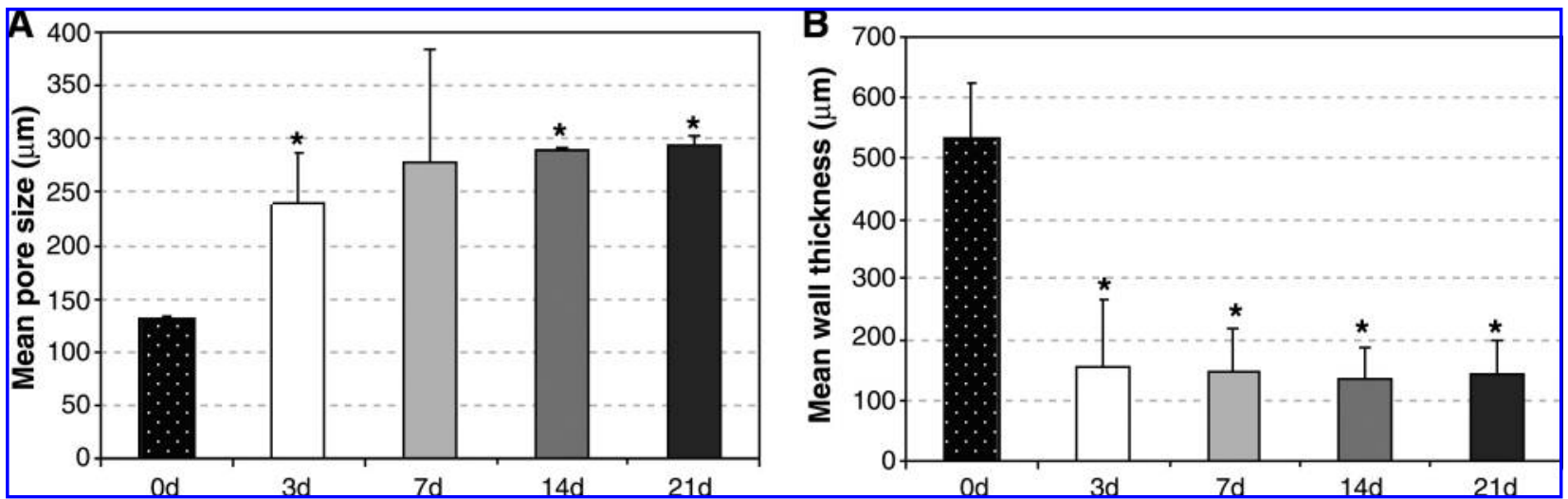

FIG. 4. Pore size (A) and wall thickness (B) of chitosan scaffolds as a function of immersion time with lysozyme (1.5 g/L). Results are expressed as means \pm standard deviation with $n=3$ for each bar. Asterisk $\left(^{*}\right)$ indicates a significant difference $(p<0.05)$ as a function of time compared with control samples. Immersion of chitosan scaffolds in lysozyme solution increased the mean pore size and decreased the mean pore wall thickness of the scaffolds with time.

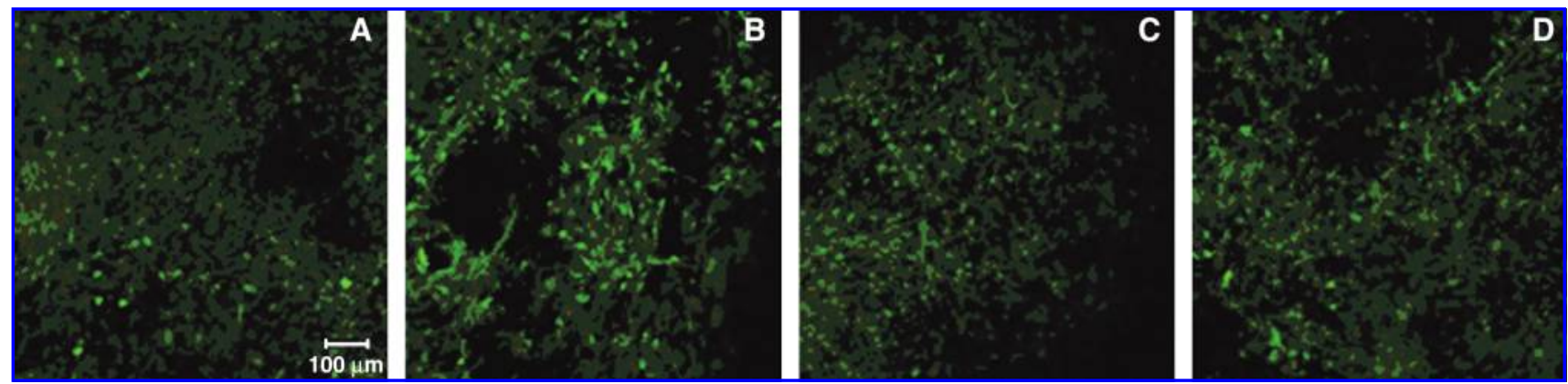

FIG. 5. Laser scanning confocal microscope images of cells stained with calcein-AM at the top surface of scaffolds after 8 days: (A) chitosan (Ch), (B) CaP-coated chitosan scaffolds with incorporated lysozyme, (C) chitosan/starch (CS), and (D) $\mathrm{CaP}$-coated chitosan/starch scaffolds with incorporated lysozyme. All images obtained 45 min after incubation with calceinAM using a 10× APO objective. The scale bar is $100 \mu \mathrm{m}$ and applies to all images. Rat MSCs adhered to all scaffold formulations and maintained viability after 8 days of culture, with no differences observed among the groups. 
concentration of similar enzymes previously incorporated into biomimetic CaP coatings ${ }^{22}$ and the concentration of lysozyme incorporated into the $\mathrm{CaP}$ coatings in the present study. ${ }^{39,40}$ As observed in Figure 1, chitosan scaffolds were degraded gradually as a function of immersion time in lysozyme solution $(1.5 \mathrm{~g} / \mathrm{L})$. The weight loss (\%) after $3,7,14$, and 21 days was $11.6 \pm 0.5,14.9 \pm 0.4,15.6 \pm 0.4$, and $18.2 \pm 1.1$, respectively (Fig. 1). This gradual weight loss is confirmed by images obtained by $\mu-\mathrm{CT}$, where an almost nonporous structure before degradation was observed (Fig. 2A) with a significant increase in porosity after different time periods, being obvious after 14 days (Fig. 2D) and 21 days (Fig. 2E). These results demonstrate the ability of lysozyme to induce degradation and subsequent formation of pores into chitosan scaffolds. Enzyme-independent starch erosion might have contributed to the scaffold degradation observed in the present study. Although minimal enzymeindependent erosion was expected, the erosion of starch from chitosan/starch scaffolds in the absence of lysozyme was not examined in the present study and will be investigated in future studies. As the present study established that lysozyme in solution at a concentration similar to the concentration incorporated into the $\mathrm{CaP}$ coatings enhances pore formation and interconnectivity with time, future studies will examine the degradation of CaP-coated chitosan scaffolds with incorporated (i.e., entrapped) lysozyme.

Upon analyzing the images of chitosan (Ch) scaffolds obtained by $\mu-\mathrm{CT}$, it is possible to see the increase of porosity as a function of immersion time (Fig. 2). The control scaffolds (before degradation) presented $\sim 5 \%$ porosity. According to Figure $3 \mathrm{~A}$ lysozyme enhanced the porosity of the scaffolds as a function of degradation time. After different time points, lysozyme had a significant effect on chitosan scaffolds, inducing the formation of a porous structure (Figs. 2 and 3A). Further, the interconnectivity of chitosan scaffolds increased significantly after 21 days (Fig. 3B). Moreover, the effect of lysozyme was also detected in pore size and wall thickness results (Fig. 4A, B), where a significant increase in size of pores (Fig. 4A) and a significant decrease of wall thickness (Fig. 4B) were evident compared with control scaffolds (before degradation). These results were expected since after different periods of degradation with lysozyme the formation of pores was observed, evidencing a significant increase of porosity as a function of degradation time $\sim 5-55 \%$ (Fig. $3 \mathrm{~A})$ up to 21 days, resulting in porous $3 \mathrm{D}$ structures with interconnected pores $(\sim 78 \%)$. The observed increase in scaffold porosity and pore interconnectivity could be due to an increase in the size of existing pores, the formation of new pores, or a combination of the two mechanisms. However, the exact mechanism by which the scaffold porosity and pore interconnectivity increased in the present study was not elucidated.

\section{Assays}

Confocal microscopy images after 8 days show that rat MSCs were able to adhere to all scaffold formulations and maintain their viability. Cells covered the surface of all scaffolds, and no differences can be observed among groups (Fig. 5).

Scaffold cellularity is presented in Figure 6. It should be noted that for cases in which cells were completely en-

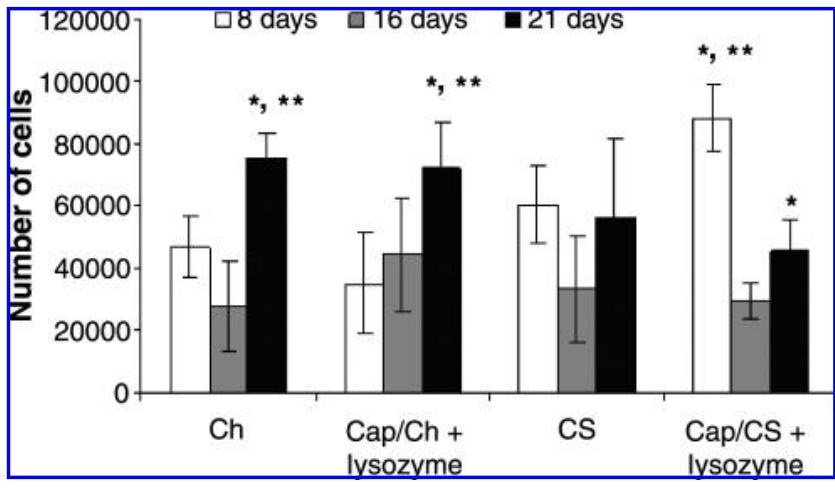

FIG. 6. Cellularity of chitosan-based scaffolds seeded with MSCs after 8, 16, and 21 days. Results are expressed as means \pm standard deviation with $n=3$ for each bar. Single asterisk $\left(^{*}\right)$ indicates a significant difference $(p<0.05)$ between conditions at the same time point. Double asterisks $\left.{ }^{* *}\right)$ indicate a significant difference $(p<0.05)$ between different time periods for each condition. An increase in cell number indicating cell proliferation was observed between days 16 and 21 for all groups. $\mathrm{Ch}$, chitosan scaffolds; $\mathrm{CaP} / \mathrm{Ch}+$ lysozyme, $\mathrm{CaP}$-coated chitosan scaffolds with incorporated lysozyme; $\mathrm{CS}$, chitosan/starch scaffolds; $\mathrm{CaP} / \mathrm{CS}+$ lysozyme, $\mathrm{CaP}$ coated chitosan/starch scaffolds with incorporated lysozyme.

trapped within ECM produced during culture, such that the DNA therein could not be fully liberated for inclusion in the lysate for assessment of cellularity, then the measured results may reflect an underestimation of the actual number of cells on/within the scaffold, especially at later time points. However, an increase in cell number indicating cell proliferation was observed between days 16 and 21 for all groups. The number of cells after 8 days was lower than was expected, except for CaP-coated chitosan/starch with incorporated lysozyme. This can be related to the small surface area of scaffolds where cells were seeded and explained by a lack of initial porosity in these scaffolds. However, after 21 days, a significant increase was observed in the number of cells for CaP-coated chitosan scaffolds with incorporated lysozyme, which corresponds to the increase in porosity as a function of time, as was shown in the degradation results (Fig. 1). During the time of culture with MSCs, it was expected that lysozyme would be able to degrade CaP-coated chitosanbased scaffolds, inducing the formation of pores by lysozyme and allowing the penetration and colonization of cells into the scaffolds, as shown in Figure 7B after 21 days.

The use of biomimetic coating methodology in this study allowed the incorporation of lysozyme that will be able to degrade chitosan-based scaffolds. It is expected that $\mathrm{CaP}$ coatings will enhance the osteoconductive properties of the resulting materials. The SEM images after 8 days show cells attached to scaffold surfaces; however, after 16 and 21 days all surfaces were completely colonized by the cells, and after 21 days mineralized matrix was observed on $\mathrm{CaP}$-coated chitosan-based scaffolds (Fig. 7A), clearly visible at high magnifications for scaffolds with incorporated lysozyme (Fig. 7B). These results corroborate the calcium content measurements, where calcium was detected after 16 and 21 days (Fig. 8). Additionally, small pores were observed in the dense layer for $\mathrm{CaP} / \mathrm{CS}+$ lysozyme at high magnifications 


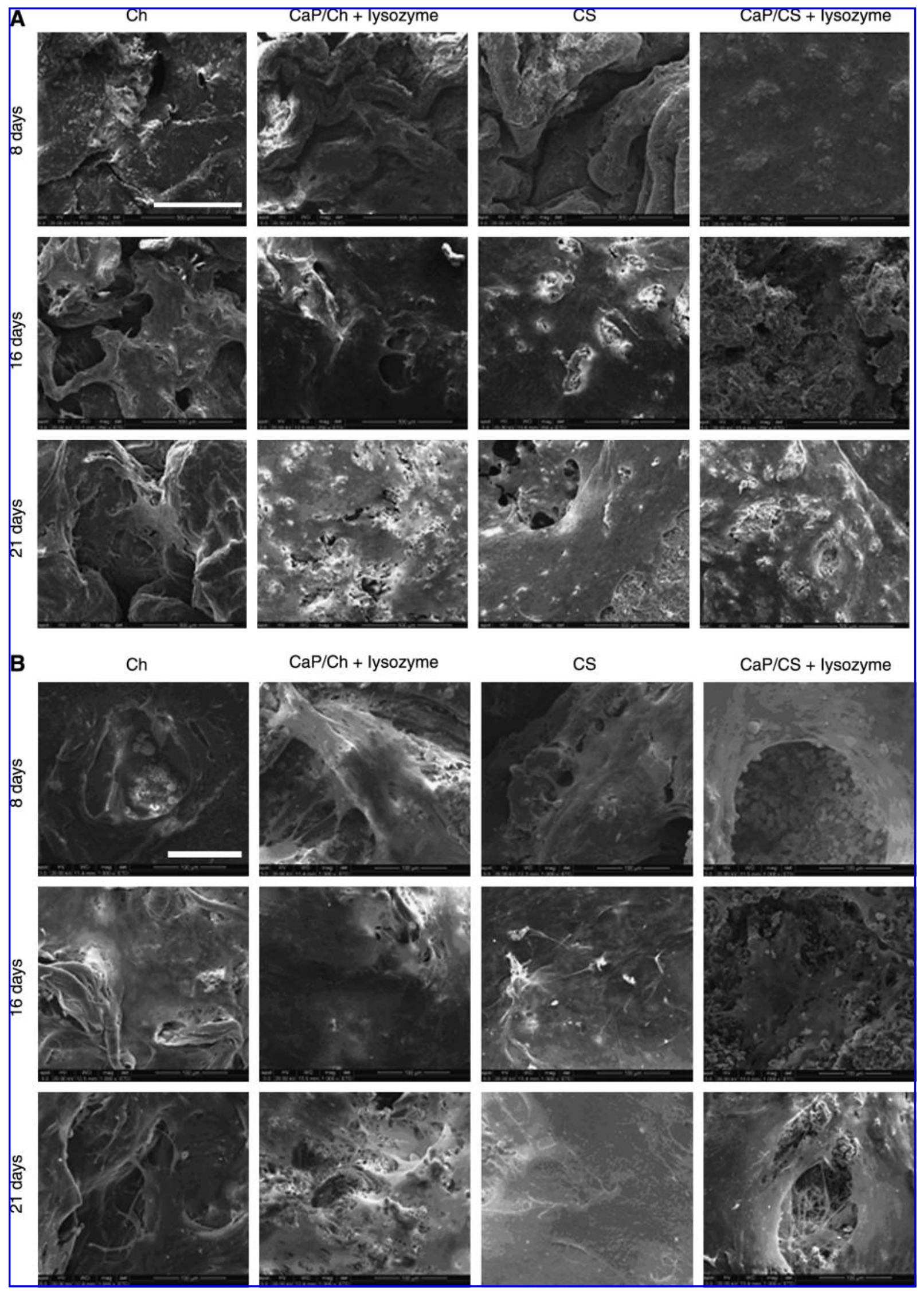

FIG. 7. (A) SEM images of the surfaces of cultured chitosan-based scaffolds after 8,16 , and 21 days. The scale bar is $500 \mu \mathrm{m}$ and applies to all images. Cells were visibly attached to scaffold surfaces for all groups after 8 days of culture, and the surfaces were completely colonized by cells after 16 and 21 days. (B) SEM images of the surfaces of cultured chitosan-based scaffolds after 8, 16, and 21 days. The scale bar is $100 \mu \mathrm{m}$ and applies to all images. These high-magnification images of the same groups represented in (A) show mineralized matrix deposition on CaP-coated chitosan-based scaffolds with incorporated lysozyme. 


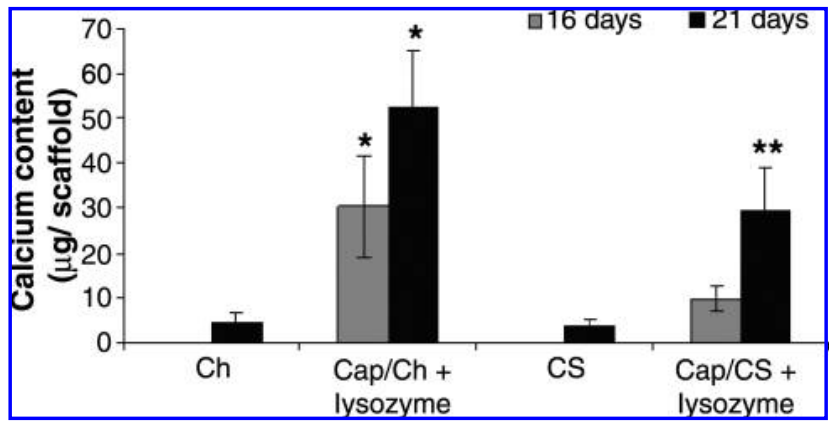

FIG. 8. Calcium content in chitosan-based scaffolds after day 16 and day 21 culture periods. Results are expressed as means \pm standard deviation with $n=3$ for each bar. Single asterisk $\left(^{*}\right)$ indicates a significant difference $(p<0.05)$ between conditions at the same time point. Double asterisks $\left({ }^{* *}\right)$ indicate a significant difference between days 16 and 21 for each condition. Calcium deposition increased with culture time for all groups, indicating the osteoblastic differentiation of the MSCs cultured upon the scaffolds. Ch, chitosan scaffolds; $\mathrm{CaP} / \mathrm{Ch}+$ lysozyme, $\mathrm{CaP}$-coated chitosan scaffolds with incorporated lysozyme; CS, chitosan/starch scaffolds; $\mathrm{CaP} / \mathrm{CS}+$ lysozyme, $\mathrm{CaP}$-coated chitosan/starch scaffolds with incorporated lysozyme.

after 8 and 21 days. These pores seem to be induced by the action of lysozyme present in $\mathrm{CaP}$ coatings (Fig. 7B).

ALP is an enzyme expressed by cells and is an early marker of osteoblastic differentiation in MSCs. ${ }^{41} \mathrm{CaP} / \mathrm{CS}+$ lysozyme and uncoated scaffolds (Ch and CS) reached their maximum measured ALP activity on day 8 and 16, respectively (Fig. 9), reflecting the early osteogenic differentiation stage of the MSCs. The highest value of ALP activity for $\mathrm{CaP} / \mathrm{Ch}+$ lysozyme was obtained at day 21 ; however, the difference between the 16th and 21st days was not statistically significant.

Calcium deposition is an indicator of the level of mineral deposition, which is a marker of the full maturation of osteoblastic cells. No calcified ECM deposition was observed at day 8 (Fig. 8). After 16 days, calcium deposition was detected for CaP-coated chitosan-based scaffolds with incorporated lysozyme. At the end of the third week, the calcium measurements indicated the deposition of significant amounts of mineralized matrix for all scaffolds, with a significant increase for CaP-coated chitosan-based scaffolds with incorporated lysozyme (Fig. 8). The amount of calcium detected on $\mathrm{CaP}$-coated scaffolds was higher for both time points of $\mathrm{CaP} / \mathrm{Ch}+$ lysozyme; however, when comparing cellularity (Fig. 6), it is apparent that the number of cells was also higher, indicating that the lower number of cells present in $\mathrm{CaP} / \mathrm{CS}+$ lysozyme also was able to produce mineralized ECM. Nevertheless, when comparing the number of cells present in these two scaffolds with the calcium content, both scaffolds had a similar effect on the cells. Indeed, comparison of cellularity results (Fig. 6) and ALP activity results (Fig. 9) reveals that the maximum ALP activity occurred at either day 16 or 21 for all groups except the $\mathrm{CaP} / \mathrm{CS}+$ lysozyme group. As ALP activity is an early marker of osteoblastic differentiation of cells, and as cellular differentiation and proliferation are inversely proportional, it follows that a minimum in cellularity at day 16 may reasonably correspond

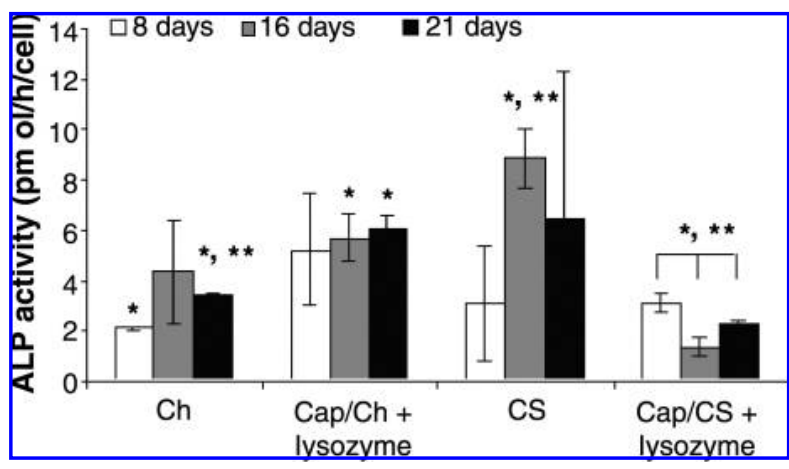

FIG. 9. ALP activity of chitosan-based scaffolds seeded with MSCs after 8, 16, and 21 days. Results are expressed as means \pm standard deviation with $n=3$ for each bar. Single asterisk $\left({ }^{*}\right)$ indicates a significant difference $(p<0.05)$ between conditions at the same time point. Double asterisk $\left({ }^{* *}\right)$ indicates a significant difference $(p<0.05)$ between 8,16 , and 21 days for each condition. $\mathrm{CaP} / \mathrm{CS}+$ lysozyme and uncoated scaffolds ( $\mathrm{Ch}$ and $\mathrm{CS}$ ) reached their maximum measured ALP activity on day 8 and 16, respectively, reflecting the early osteogenic differentiation stage of the MSCs. $\mathrm{Ch}$, chitosan scaffolds; $\mathrm{CaP} / \mathrm{Ch}+$ lysozyme, CaP-coated chitosan scaffolds with incorporated lysozyme; CS, chitosan/ starch scaffolds; CaP/CS + lysozyme, CaP-coated chitosan/ starch scaffolds with incorporated lysozyme.

with a peak in ALP activity at day 16. Further, the presence of mineralized matrix, as measured by calcium content of constructs, increased with time for all groups, indicating the osteogenic differentiation of cells in all groups, although this effect was most pronounced in the CaP-coated scaffolds with incorporated lysozyme.

\section{FTIR with ATR}

When FTIR-ATR spectra of controls (without cells) of chitosan (Ch) and chitosan/starch (CS) scaffolds were analyzed, the characteristic bands of chitosan $\left(1150-890 \mathrm{~cm}^{-1}\right)$ were detected (Fig. 10A, C). After 16 and 21 days of culture (Fig. 10A, C), these spectra showed the presence of amide bands: $1650 \mathrm{~cm}^{-1}$ (amide I) and $1542 \mathrm{~cm}^{-1}$ (amide II), which can be related to protein matrix formation. Carbonate groups at $1500-1418 \mathrm{~cm}^{-1}$ were also detected (Fig. 10A, B). These carbonate groups are related to carbonate apatite, one of the CaPs present in bone tissue, suggesting the presence of mineralized ECM. Presented in Figure 10B and 10D are the spectra of $\mathrm{CaP} / \mathrm{Ch}$ with incorporated lysozyme and $\mathrm{CaP} / \mathrm{CS}$ with incorporated lysozyme scaffolds. In FTIR-ATR spectra of the CaP-coated chitosan-based scaffolds when lysozyme was incorporated, the presence of amide I and amide II in uncultured scaffolds (controls) was detected (Fig. 10B, D). The amide I band represents the stretching vibrations of $\mathrm{C}=\mathrm{O}$ bonds in the backbone of the protein. ${ }^{42}$ Further, the amide II band arises from the combination of C-N stretching and $\mathrm{N}-\mathrm{H}$ bending vibrations of the protein backbone. ${ }^{42}$ These bands obtained in the case of the control samples could indicate the adsorption of lysozyme on the surface of chitosan and chitosan/starch scaffolds. However, an increase of intensity of these bands was observed as a function of culture time (Fig. 10B, D). As previously mentioned, these might indicate a protein matrix formation. In the spectra of control 

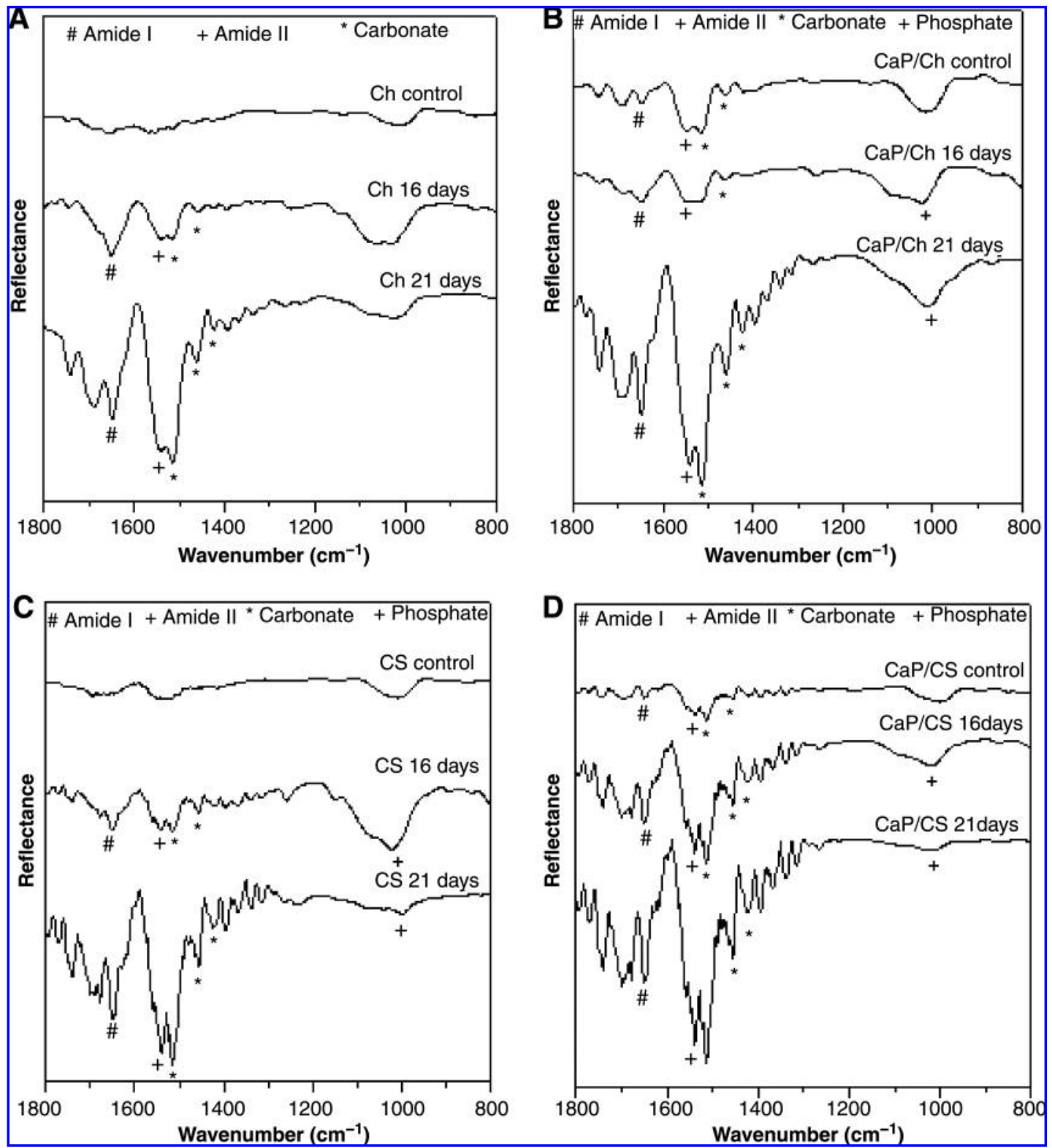

FIG. 10. FTIR-ATR of chitosan (A), CaP/Ch + lysozyme (B), chitosan/starch (C), and CaP/CS + lysozyme (D) scaffolds before and after 16 and 21 days of culture with rat MSCs. Increases in the amide, carbonate, and phosphate bands indicate increased deposition of mineralized ECM with time.

scaffolds (Fig. 10B, D), carbonate bands $\left(1500-1418 \mathrm{~cm}^{-1}\right.$ ) were also detected, which are associated with $\mathrm{CaP}$ coating on the surface of the scaffolds. In addition, these bands became more intense with culture time, suggesting the formation of mineralized ECM. However, phosphate bands (1190$976 \mathrm{~cm}^{-1}$ ) were not clearly detected, which was likely related to overlapping with the polysaccharide ring band of chitosan $\left(1150-890 \mathrm{~cm}^{-1}\right)$.

FTIR-ATR results confirm the results of calcium measurements, clearly suggesting protein matrix formation and the presence of mineralized ECM. Further, these results suggest that the mineral formed by rat MSCs cultured for 16 and 21 days is carbonate apatite mineral similar to the major mineral component of bone. Future studies will build upon the results from the present study, which demonstrates that lysozyme has an effect on pore formation in chitosan scaffolds and osteogenic differentiation of MSCs, and will focus upon the use of different concentrations of lysozyme and characterization of the actual lysozyme content of the scaffolds.

\section{Conclusions}

The degradation studies performed with lysozyme using similar concentration to those incorporated into $\mathrm{CaP}$ coatings showed the formation of pores as a function of immersion time, indicating an increase of porosity $\sim 5-55 \%$ up to 21 days. Lysozyme had a significant effect on chitosan scaffolds resulting in porous 3D structures with interconnected pores $(\sim 78 \%)$. On the basis of these studies it was concluded that $\mathrm{CaP}$ biomimetic coatings with incorporated lysozyme 
enhance osteogenic differentiation of rat MSCs. Therefore, the use of $\mathrm{CaP}$ biomimetic coating strategy with incorporated lysozyme on the surface of chitosan-based scaffolds provides an improved environment for rat MSC differentiation along the osteogenic lineage when compared with uncoated scaffolds, as was shown by calcium deposition measurements and FTIR-ATR results, indicating the production of mineralized ECM. The demonstrated ability of these novel surfacecoated scaffolds to form an interconnected pore structure in situ coupled with the positive effect of these scaffolds on the osteoblastic differentiation of MSCs and mineralized matrix production clearly illustrate the strong potential of these scaffolds for application in bone tissue engineering strategies.

\section{Acknowledgments}

The authors would like to acknowledge Dr. Serena Danti. This work was supported by the European NoE EXPERTISSUES (NMP3-CT-2004-500283), the European STREP HIPPOCRATES (NMP3-CT-2003-505758), and the Portuguese Foundation for Science and Technology (FCT) through POCTI and/or FEDER programs. This work was also supported by a grant from the National Institutes of Health (NIH; R01 DE15164) (A.G.M.) and a Bioengineering Research Partnership with the Baylor College of Medicine through the National Institute of Biomedical Imaging and Bioengineering (NIH Grant 5 R01 EB005173-02). F.K.K. is supported by a training fellowship from the Keck Center Nanobiology Training Program of the Gulf Coast Consortia (NIH Grant 5 T90 DK070121-03).

\section{Disclosure Statement}

No competing financial interests exist.

\section{References}

1. Langer, R., and Vacanti, J.P. Tissue engineering. Science 260, 920, 1993.

2. Atala, A. Engineering tissues, organs and cells. J Tissue Eng Regen Med 1, 83, 2007.

3. Bruder, S.P., and Fox, B.S. Tissue engineering of bone. Cell based strategies. Clin Orthop Relat Res, S68, 1999.

4. Salgado, A.J., Coutinho, O.P., and Reis, R.L. Bone tissue engineering: state of the art and future trends. Macromol Biosci 4, 743, 2004.

5. Hutmacher, D.W., Schantz, J.T., Lam, C.X.F., Tam, K.C., and Lim, T.C. State of the art and future directions of scaffoldbased bone engineering from a biomaterials perspective. J Tissue Eng Regen Med 1, 245, 2007.

6. Martins, A.M., Santos, M.I., Azevedo, H.S., Malafaya, P.B., and Reis, R.L. Natural origin scaffolds with in situ pore forming capability for bone tissue engineering applications. Acta Biomater, 4, 1637, 2008.

7. Tuzlakoglu, K., Alves, C.M., Mano, J.F., and Reis, R.L. Production and characterization of chitosan fibers and 3-D fiber mesh scaffolds for tissue engineering applications. Macromol Biosci 4, 811, 2004.

8. Malafaya, P.B., Pedro, A.J., Peterbauer, A., Gabriel, C., Redl, H., and Reis, R.L. Chitosan particles agglomerated scaffolds for cartilage and osteochondral tissue engineering approaches with adipose tissue derived stem cells. J Mater Sci Mater Med 16, 1077, 2005.
9. Oliveira, J.M., Rodrigues, M.T., Silva, S.S., Malafaya, P.B., Gomes, M.E., Viegas, C.A., Dias, I.R., Azevedo, J.T., Mano, J.F., and Reis, R.L. Novel hydroxyapatite/chitosan bilayered scaffold for osteochondral tissue-engineering applications: scaffold design and its performance when seeded with goat bone marrow stromal cells. Biomaterials 27, 6123, 2006.

10. Chatelet, C., Damour, O., and Domard, A. Influence of the degree of acetylation on some biological properties of chitosan films. Biomaterials 22, 261, 2001.

11. Klokkevold, P.R., Vandemark, L., Kenney, E.B., and Bernard, G.W. Osteogenesis enhanced by chitosan (poly-N-acetyl glucosaminoglycan) in vitro. J Periodontol 67, 1170, 1996.

12. Lahiji, A., Sohrabi, A., Hungerford, D.S., and Frondoza, C.G. Chitosan supports the expression of extracellular matrix proteins in human osteoblasts and chondrocytes. I Biomed Mater Res 51, 586, 2000.

13. Pound, J.C., Green, D.W., Chaudhuri, J.B., Mann, S., Roach, H.I., and Oreffo, R.O. Strategies to promote chondrogenesis and osteogenesis from human bone marrow cells and articular chondrocytes encapsulated in polysaccharide templates. Tissue Eng 12, 2789, 2006.

14. Peluso, G., Petillo, O., Ranieri, M., Santin, M., Ambrosio, L., Calabro, D., Avallone, B., and Balsamo, G. Chitosanmediated stimulation of macrophage function. Biomaterials 15, 1215, 1994.

15. Muzzarelli, R.A. Human enzymatic activities related to the therapeutic administration of chitin derivatives. Cell Mol Life Sci 53, 131, 1997.

16. Varum, K.M., Myhr, M.M., Hjerde, R.J., and Smidsrod, O. In vitro degradation rates of partially $\mathrm{N}$-acetylated chitosans in human serum. Carbohydr Res 299, 99, 1997.

17. Tomihata, K., and Ikada, Y. In vitro and in vivo degradation of films of chitin and its deacetylated derivatives. Biomaterials 18, 567, 1997.

18. Mendes, S.C., Reis, R.L., Bovell, Y.P., Cunha, A.M., van Blitterswijk, C.A., and de Bruijn, J.D. Biocompatibility testing of novel starch-based materials with potential application in orthopaedic surgery: a preliminary study. Biomaterials 22, 2057, 2001.

19. Marques, A.P., Reis, R.L., and Hunt, J.A. The biocompatibility of novel starch-based polymers and composites: in vitro studies. Biomaterials 23, 1471, 2002.

20. Salgado, A.J., Coutinho, O.P., Reis, R.L., and Davies, J.E. In vivo response to starch-based scaffolds designed for bone tissue engineering applications. J Biomed Mater Res A 80, 983, 2007.

21. Azevedo, H.S., Gama, F.M., and Reis, R.L. In vitro assessment of the enzymatic degradation of several starch based biomaterials. Biomacromolecules 4, 1703, 2003.

22. Azevedo, H.S., Leonor, I.B., Alves, C.M., and Reis, R.L. Incorporation of proteins and enzymes at different stages of the preparation of calcium phosphate coatings on a degradable substrate by a biomimetic methodology. Mater Sci Eng C-Bio S 25, 169, 2005.

23. Reis, R.L., and Cunha, A.M. Starch and starch based thermosplastic. In: Jurgen, K.H., Buschow, R., Cahn, W., Flemings, M.C., Ilschner, B., Kramer, E.J., and Mahajan, S., eds. Encyclopedia of Materials Science and Technology. Amsterdam: Pergamon-Elsevier Science, 2001, pp. 8810-8816.

24. Azevedo, H.S., and Reis, R.L. Understanding the enzymatic degradation of biodegradable polymers and strategies to control their degradation rate. In: Reis, R.L., and Roman, J.S., eds. Biodegradable Systems in Tissue Engineering and Regenerative Medicine, Boca Raton: CRC Press, pp. 177-201, 2005. 
25. Liu, Y., Hunziker, E.B., Randall, N.X., de Groot, K., and Layrolle, P. Proteins incorporated into biomimetically prepared calcium phosphate coatings modulate their mechanical strength and dissolution rate. Biomaterials 24, 65, 2003.

26. Liu, Y., Hunziker, E.B., Layrolle, P., De Bruijn, J.D., and De Groot, K. Bone morphogenetic protein 2 incorporated into biomimetic coatings retains its biological activity. Tissue Eng 10, 101, 2004.

27. Azevedo, H.S., Leonor, I.B., and Reis, R.L. Incorporation of proteins with different isoelectric points into biomimetic Ca-P coatings: a new approach to produce hybrid coatings with tailored properties. Key Eng Mater 309, 755, 2006.

28. Ohtsuki, C., Kamitakahara, M., and Miyazaki, T. Coating bone-like apatite onto organic substrates using solutions mimicking body fluid. J Tissue Eng Regen Med 1, 33, 2007.

29. Barrere, F., van der Valk, C.M., Meijer, G., Dalmeijer, R.A., de Groot, K., and Layrolle, P. Osteointegration of biomimetic apatite coating applied onto dense and porous metal implants in femurs of goats. J Biomed Mater Res B Appl Biomater 67, 655, 2003.

30. Habobovic, P., and de Groot, K. Osteoinductive biomaterialsproperties and relevance in bone repair. J Tissue Eng Regen Med 1, 25, 2007.

31. Abe, Y., Kokubo, T., and Yamamuro, T. Apatite coating on ceramics, metals and polymers utilizing a biological process. J Mater Sci Mater Med 1, 233, 1990.

32. Reis, R.L., Cunha, A.M., Fernandes, M.H., and Correia, R.N. Treatments to induce the nucleation and growth of apatitelike layers on polymeric surfaces and foams. J Mater Sci Mater Med 8, 897, 1997.

33. Martins, A.M., Salgado, A.J., Azevedo, H.S., Leonor, I.B., and Reis, R.L. Lysozyme incorporation in biomimetic coated chitosan scaffolds: development and behaviour in contact with osteoblastic-like cells. Tissue Eng 12, 1018, 2006.

34. Hacker, M., Ringhofer, M., Appel, B., Neubauer, M., Vogel, T., Young, S., Mikos, A.G., Blunk, T., Gopferich, A., and Schulz, M.B. Solid lipid templating of macroporous tissue engineering scaffolds. Biomaterials 28, 3497, 2007.

35. Lu, J.X., Flautre, B., Anselme, K., Hardouin, P., Gallur, A., Descamps, M., and Thierry, B. Role of interconnections in porous bioceramics on bone recolonization in vitro and in vivo. J Mater Sci Mater Med 10, 111, 1999.
36. Otsuki, B., Takemoto, M., Fujibayashi, S., Neo, M., Kokubo, T., and Nakamura, T. Pore throat size and connectivity determine bone and tissue ingrowth into porous implants: three-dimensional micro-CT based structural analyses of porous bioactive titanium implants. Biomaterials 27, 5892, 2006.

37. Shi, X., Sitharaman, B., Pham, Q.P., Liang, F., Wu, K., Edward Billups, W., Wilson, L.J., and Mikos, A.G. Fabrication of porous ultra-short single-walled carbon nanotube nanocomposite scaffolds for bone tissue engineering. Biomaterials 28, 4078, 2007.

38. Maniatopoulos, C., Sodek, J., and Melcher, A.H. Bone formation in vitro by stromal cells obtained from bone marrow of young adult rats. Cell Tissue Res 254, 317, 1988.

39. Datta, N., Holtorf, H.L., Sikavitsas, V.I., Jansen, J.A., and Mikos, A.G. Effect of bone extracellular matrix synthesized in vitro on the osteoblastic differentiation of marrow stromal cells. Biomaterials 26, 971, 2005.

40. Holtorf, H.L., Jansen, J.A., and Mikos, A.G. Ectopic bone formation in rat marrow stromal cell/titanium fiber mesh scaffold constructs: effect of initial cell phenotype. Biomaterials 26, 6208, 2005.

41. Toquet, J., Rohanizadeh, R., Guicheux, J., Couillaud, S., Passuti, N., Daculsi, G., and Heymann, D. Osteogenic potential in vitro of human bone marrow cells cultured on macroporous biphasic calcium phosphate ceramic. J Biomed Mater Res 44, 98, 1999.

42. Xie, J., Riley, C., Kumar, M., and Chittur, K. FTIR/ATR study of protein adsorption and brushite transformation to hydroxyapatite. Biomaterials 23, 3609, 2002.

Address correspondence to: Antonios G. Mikos, Ph.D.

Department of Bioengineering Rice University

MS-142, P.O. Box 1892

Houston, TX 77251-1892

E-mail: mikos@rice.edu

Received: January 14, 2008

Accepted: November 25, 2008

Online Publication Date: January 20, 2009 
